\title{
El razonamiento proporcional en educación básica
}

$\frac{1}{2}$
$\frac{2}{0}$
$\frac{0}{0}$
$\frac{0}{3}$
$\frac{0}{2}$

Proportional reâsoning in basic education

(c) (i) () (2)

Cristianne Butto Zarzar Joaquín Delgado Fernández

Deysi Calderón Araujo Aldo Bazán Ramírez
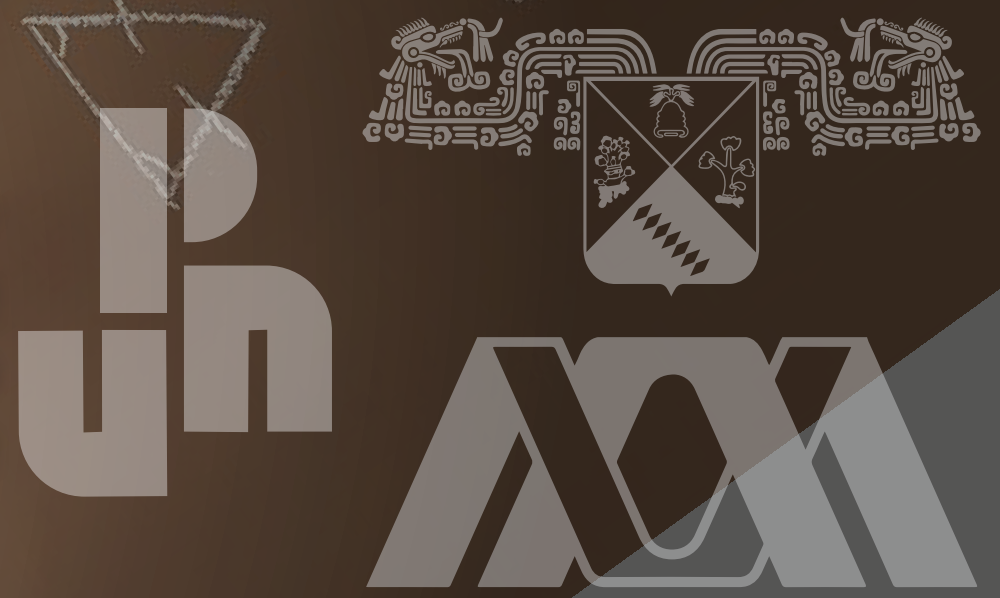


\section{HORIZONTES PEDAGÓGICOS}

ID: Title:

Título:

0123-8264.hop.21204

Proportional reasoning in basic education

\section{Iiento proporcional en} educación básica

\section{Alt Title / Título alternativo:}

[en]: $\quad$ Proportional reasoning in basic education

[es]: $\quad$ El razonamiento proporcional en educación básica

Author (s) / Autor (es):

Butto Zarzar, Delgado Fernández, Calderón Araujo, \& Bazán Ramírez

\section{Keywords / Palabras Clave: \\ [en]: \\ proportional reasoning; validation of an instrument; basic education; algebraic thinking; problem solving; levels of achievement; learning \\ [es]: $\quad$ razonamiento proporcional; validación de un instrumento; educación básica; pensamiento algebraico; resolucion de problemas; niveles de logro; aprendizaje}

Submited:

2019-10-18

\section{Resumen}

Este artículo de investigación es el resultado de un proyecto de investigación sobre el pensamiento algebraico temprano en entornos tecnológicos de aprendizaje y se reportan resultados sobre el razonamiento proporcional como una ruta de acceso

al pensamiento algebraico temprano. El estudio fue realizado con 109 estudiantes de educación básica de escuelas públicas, del Estado de México y de la

Ciudad de México y uno de los objetivos del estudio fue validar un instrumento sobre el razonamiento proporcional. Para ello, se utilizó una metodología mixta, un estudio de tipo observacional, longitudinal y comparativo con tres etapas: 1. Evaluación inicial sobre el razonamiento proporcional, 2.Validación de un instrumento sobre el razonamiento proporcional,

3. Entrevista clínica abierta. Los resultados revelan que los alumnos logran comprender ideas de proporcionalidad aritmética y geométrica y variación proporciona, y comprenden los problemas que involucran la relación funcional, como una consecuencia del tránsito del pensamiento aditivo al multiplicativo. En lo que respecta al instrumento de evaluación, se propuso un modelo hipotético de validez convergente y divergente de constructo en la medición del razonamiento proporcional y el modelo resultante obtuvo aceptables indicadores

prácticos de bondad de ajuste y coeficientes de confiabilidad altos. Además el instrumento mostró tener validez convergente y divergente de constructo.

Finalmente, podemos concluir que el razonamiento proporcional es una ruta viable hacia el pensamiento algebraico, a pesar de que los estudiantes no han desarrollado aún todas las estructuras cognitivas y matemáticas para comprender la complejidad del pensamiento proporcional, los resultados de este estudio permitieron dilucidar etapas tempranas en el desarrollo cognitivo de los niños con tareas y enfoques pertinentes.

\section{Abstract}

This research article is the result of a research project on early algebraic thinking in technological learning environments and results on proportional reasoning are reported as a path to early algebraic thinking. The study was carried out with 109 basic education students from public schools, the State of Mexico and Mexico City and one of the objectives of the study was to

validate an instrument on proportional reasoning. For this, a mixed methodology was used, an observational, longitudinal and comparative study with three stages: 1. Initial evaluation on proportional reasoning, 2. Validation of an instrument on proportional reasoning, 3. Open clinical interview. The results reveal that students manage to understand ideas of arithmetic and geometric proportionality and variation provides, and understand the problems that involve the

functional relationship, as a consequence of the transition from additive to multiplicative thinking. With regard to the evaluation instrument, a hypothetical model of convergent and divergent construct validity was proposed in the measurement of proportional reasoning and the resulting model obtained acceptable practical indicators of goodness of fit and high reliability coefficients. In addition, the instrument showed convergent and divergent construct validity. Finally, we can conclude that proportional reasoning is a viable route to algebraic thinking, although the students have not yet developed all the cognitive and mathematical structures to understand the complexity of proportional thinking, the results of this study allowed us to elucidate early stages in the cognitive development of children with relevant tasks and approaches.

\begin{abstract}
Dra Cristianne Butto Zarzar, MEd Bed
\end{abstract}
ORCID: 0000-0001-8913-2832

\section{Source | Filiacion:}

Universidad Pedagógica Nacional Ajusco

BIO:

Doctora en Ciencias Especialidad en

Matemática Educativa, Cinestav [mx];

Maestria en Psicología Cognitiva,

Universidade Federal de Pernambuco [br],

Licenciatura en Pedagogía, Universidade

Federal de Pernambuco [br]; Académica

Investigadora Titular, Universidad

Pedagógica Nacional Unidad Ajusco [mx]

\section{City | Ciudad:}

Mexico DF [mx]

\section{e-mail:}

cristianne@upn.mx
Dr Joaquín Delgado Fernández

ORCID:

0000-0002-7316-6672

Dr Aldo Bazán Ramírez, Psi

ORCID:

0000-0002-0229-4431

\section{Source | Filiacion:}

Universidad Autónoma Metropolitana

BIO:

Profesor Titular, Departamento de

Matemática - UAM-Iztapala

\section{City | Ciudad:}

México DF [mx]

\section{e-mail:}

joaquin.delgado.f@gmail.com

\section{Source | Filiacion:}

Universidad Autónoma del Estado de Morelos

\section{BIO:}

Doctor en Psicología, UNAM [mx]

Psicologo, Universidad Nacional Federico Villareal [pe]

Centro de Investigación Transdisciplinar en Psicología - Universidad Autónoma del Estado de Morelos

\section{City | Ciudad:}

Cuernavaca $[\mathrm{mx}]$

\section{e-mail:}

abazanramirez@gmail.com
Lic Deysi Calderón Araujo, Psi

\section{Source | Filiacion:}

Universidad Pedagógica Nacional Ajusco

BIO:

Licenciada en psicología educativa,

Universidad Pedagógica Nacional Ajusco, México

\section{City | Ciudad:}

Mexico DF $[\mathrm{mx}]$

\section{e-mail:}

ximdan03@hotmail.com 


\section{El razonamiento proporcional en educación básica Proportional reasoning in basic education Cristianne Butto Zarzar Joaquín Delgado Fernández Deysi Calderón Araujo Aldo Bazán Ramírez}

\section{Introducción}

Razón y proporción ha sido uno de los temas más investigados en el campo de la educación matemática y existe una literatura muy amplia. Este tema ha sido estudiado por diversos autores, por ejemplo, Inhelder y Piaget (1958), describieron el razonamiento proporcional, y argumentaron que dicho razonamiento se ubica en la etapa de las operaciones formales y argumenta que éste razonamiento aparece a medida que el niño se aproxima a la adolescencia. El autor comenta que el razonamiento proporcional, la habilidad de formular hipótesis y trabajar con un cierto número de variables es una característica que revela que el estudiante se encuentra en la etapa del razonamiento formal.

Piaget también propone un seguimiento de las etapas del desarrollo cognitivo, hasta llegar al desarrollo de las operaciones formales; y comenta que es importante que el estudiante comprenda los fundamentos de los temas de razón y proporción. En sus experimentos señala que el niño adquiere la identidad cualitativa antes que la conservación cuantitativa y hace una distinción entre comparaciones cualitativas y la verdadera cuantificación. Para el referido autor, la noción de proporción empieza de manera cualitativa y lógica, antes de que se estructure cuantitativamente. Este autor comenta que, entre los 11 y 12 años, el niño percibe la presencia de la noción de las proporciones en diferentes ámbitos, por ejemplo, las proporciones espaciales. 


\section{El razonamiento proporcional en educación básica}

Sin embargo, muchos teóricos contemporáneos piensan que Piaget ha sido fuertemente criticado por sus ideas concernientes a la naturaleza cualitativa del desarrollo cognitivo, pues subestimó las capacidades de los niños de corta edad. Muchos investigadores creen el niño posee la habilidad para resolver problemas de niveles cognitivos superiores, pero la habilidad verbal aún no está completamente desarrollada para probar la presencia o la ausencia de los conceptos básicos.

Spinillo (1990), Spinillo \& Bryant, (1991 y 1993), comentan que el razonamiento proporcional no es una experiencia propia de las operaciones formales y puede ser desarrollado en niños de menor edad. Estos autores ponen en evidencia los estudios realizados con niños de 4 a 6 años de edad, quienes comprenden la idea de mitad, y son capaces de adquirir un juicio perceptual al empezar a comparar razones de cantidad antes de tener cualquier experiencia con razones numéricas y que además el tipo de tareas también hace una diferencia importante.

Esto indica que, por otra parte, que el juicio perceptual (geométrico) es una habilidad que puede ser desarrollada a temprana edad. Otro aspecto está relacionado con los elementos que perjudican el desempeño y entendimiento de las tareas propuestas a los niños, aquí destaca el lenguaje utilizado y el contexto de los problemas presentados. El propio Piaget describió estadios tempranos en el pensamiento y los denominó de estadio intermediario, utilizado en las correspondencias cualitativas y seriaciones, y concluye que puede haber un entendimiento temprano de algunos conceptos matemáticos, como el de proporcionalidad. Por otra parte, Butto (2005) señala que entre las réplicas a los estudios de Piaget se encuentran los estudios de Lunzer y Pumfrey (1966) los cuales observaron que los alumnos son capaces de registrar razones como (1:1, 2:1, y 3:1) de manera fácil al resolver los problemas de manera aditiva.

Al respecto, English y Halford (1995) comentan que una de las particularidades esenciales del razonamiento proporcional incluye recomendaciones de 20 orden, es decir, relaciones entre dos cantidades directamente claras. Perspectiva que defiende que la fase temprana del razonamiento proporcional en los niños incluye un razonamiento aditivo, de la forma $a-b=c-d$.

Entretanto, el trabajo de Lesh (1988) registra como indicador del uso del razonamiento proporcional; un razonamiento destacado, que los niños usan en varias tareas multiplicativas, que son característicamente de una ecuación de tipo, especialmente cuando tienen una solución algorítmica. Por su parte, Raspetti (2003), menciona, que el aprendizaje de la noción de proporción no es simple y requiere del alumno una gama de situaciones de diferente complejidad numérica y el tipo de magnitudes relacionadas; y que, por lo tanto, esto se convierte en un obstáculo para la comprensión de este contenido escolar.

Lunzer, Harrison, y Davey (1973) comentan que es probable que este tipo de relaciones sean enseñadas adecuadamente, pero que no son integradas directamente a nuevos problemas, sino hasta que las capacidades de razonamiento del sujeto hayan alcanzado un nivel de desarrollo más avanzado. El autor comenta sobre un conjunto de tareas con niños de 6 a 14 años, y describe las estrategias usadas, y especifica las diferentes demandas hechas por distintas razones. Los niños de todas las edades tienden a resolver de manera aditiva los problemas presentados, aunque estos problemas sugieran métodos multiplicativos.

En los estudios concernientes al campo educativo, reportan dificultades en cuanto a los procedimientos y estrategias al trabajar con problemas vinculados con el concepto de proporcionalidad, podemos señalar a Vergnaud (1983); Riccó y Rouchier (1979); Pluvinage y Dupuis (1981); Sokona (1989); además interesa hacer referencia a estudios como el de Winch (citado en Karplus, Pulos y Stage, 1983), quién estudió la habilidad de los estudiantes de primaria para resolver correctamente problemas de proporción y encuentra que los estudiantes utilizan métodos de solución: 1) La búsqueda del valor unitario, que consiste en encontrar el valor correspondiente a la unidad; en ella subyace la pregunta: ¿Cuántos para uno? y se accede a dicho valor por medio de una división, 2) El razonamiento cualitativo, que se basa en comparaciones como mayor que, más que o igual que, sin llegar a una cuantificación de las mismas.

El primer método de la búsqueda del valor unitario es el más utilizado, esto lleva a expresar la siguiente hipótesis: Los problemas en los que se hace mención explícita de las cantidades unitarias son más sencillos que aquellos en los que no hace referencia de dichas cantidades. Noelting (1980) se refiere a problemas de comparación, dos de razones enteras y dos de razones fraccionarias, y cuatro problemas de valor perdido con dos razones enteras y dos fraccionarias. Lo hace en dos grupos, uno con instrucción y el otro sin instrucción. En su estudio sobre el jugo de naranja indica que sus resultados revelaron que los estudiantes mostraron mayormente el uso de estrategias aditivas. El grupo de estudiantes que no había recibido instrucción escolar sobre proporcionalidad presentaban un razonamiento aditivo; Los problemas de comparación eran resueltos más fácilmente que los problemas de valor ausente, y los de problemas de razones fraccionarias eran más difíciles que los de razones enteras. Para la autora, una posible explicación para estos resultados es que los estudiantes, independientemente de haber recibido instrucción escolar o no, en la vida diaria mantienen contacto con problemas de comparación y en la mayoría de las veces utilizan razones enteras.

Noelting (1980), propone la teoría de la reestructuración adaptativa para permitir la transición del primer estadio al segundo (estadio temprano al intermedio) y, para analizar el tipo de comparación que los sujetos hacen cuando resuelven un problema, comparan el jugo de limón y el agua en cada primer recipiente y, comparando esas dos relaciones, seleccionan dos jugos: de limón y de agua, formando así el abordaje denominado entre estrategias; además estudia el razonamiento proporcional con problemas de comparación numérica, estableciendo razones completas sin requerir en su solución necesariamente una respuesta numérica, pues los niños deben comparar las razones de acuerdo con el dibujo mostrado:

\section{Gráfico 1}

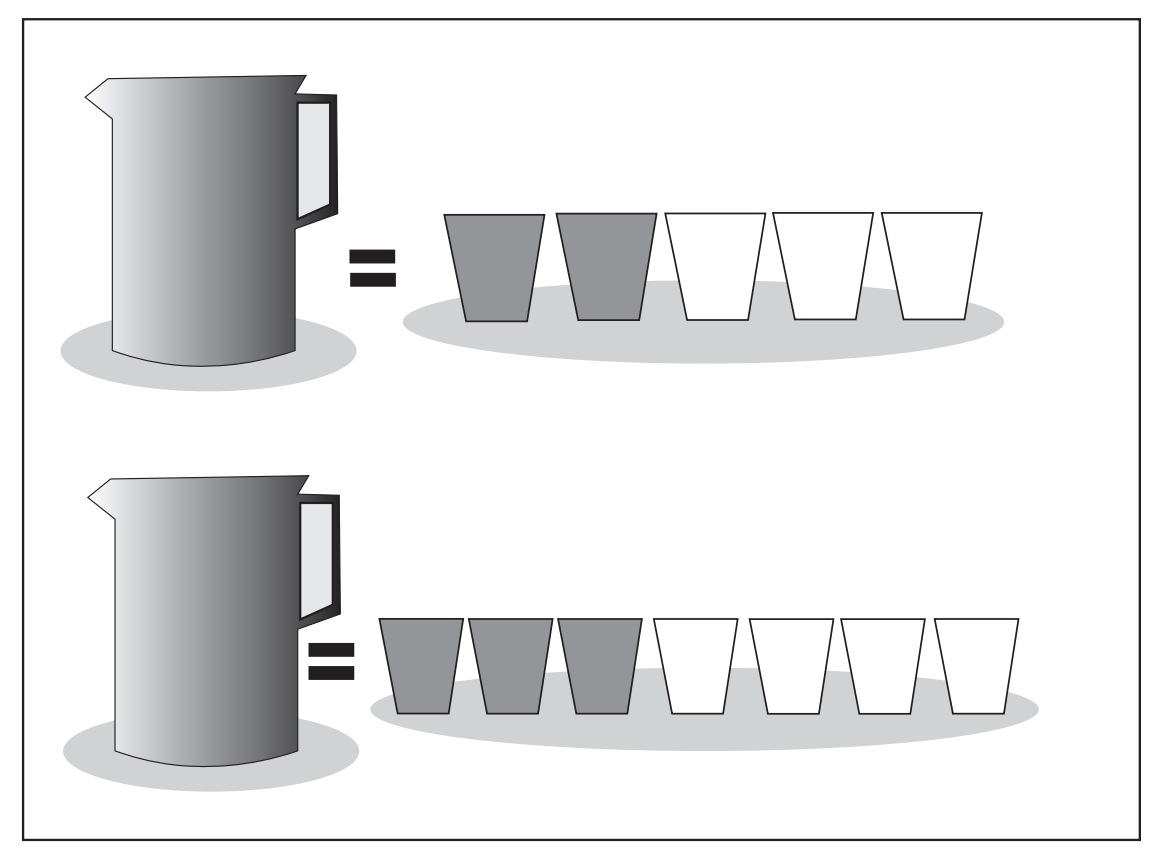

Tomado de Noelting (1980

En esta tarea, los alumnos debían responder ¿cuál jarra contiene la limonada con un sabor más fuerte o si las dos tienen el mismo sabor? Los resultados del estudio revelan que los estudiantes tienen un mejor desempeño: cuando una cantidad de la razón completa es 
un múltiplo entero, además los estudiantes usan estrategias aditivas y se observa que, en los problemas de comparación, los estudiantes presentan dificultades.

Tournaire y Pulus (1985), comentan sobre las diversas metodologías y tareas que utilizan los alumnos con el razonamiento proporcional. Estos autores mencionan que los estudiantes usan dos pares de métodos: la comparación contra valores, y explicaciones con una sola respuesta. Las tareas son variadas y se hicieron intervenciones individuales. En cada método, se identifican numerosos pensamientos utilizados en el estudio de razonamiento proporcional; también se distinguen categorías como tareas físicas, problemas de razón, problemas de mezcla y tareas de probabilidad; en todas ellas varía el contexto, y hay tipos de estrategias más utilizadas por los estudiantes, y son: variación de las estrategias, estrategias desfavorables, desarrollo de una secuencia de estrategias.

Por su parte Carraher, Schliemann, Carraher y Ruiz (1986) investigaron el desarrollo de la concepción de cantidades medidas por razones con estudiantes de primaria y ler año de secundaria. Los estudiantes fueron expuestos a dos tipos de contenidos: problemas de compra y venta y problemas de velocidad. En la primera actividad (problemas de compra y venta) el entrevistador les solicitaba a los estudiantes que indicaran, entre dos compras, cuál era la mejor y luego, les pedía una justificación de la respuesta. En la segunda (problemas de velocidad), el estudiante disponía de diversas medidas de tiempo y distancia recorridas por dos autos que eran movidos por el entrevistador, y debía indicar si los dos autos los habían movidos a la misma velocidad o no.

A partir de los resultados obtenidos en las dos actividades, revelaron una influencia de la instrucción escolar en el desempeño de los estudiantes en la actividad de velocidad. Ya en la actividad de compra y venta no se observó ningún efecto de la instrucción escolar.

Karplus y Peterson (1970), categorizaron las respuestas de los niños a partir del nivel de comprensión. El estudio sobre el señor bajo y el señor alto con una representación con clips: no todos los niños tienen una estrategia de tipo aditiva y que este pensamiento está fuertemente influenciado por la instrucción recibida en las escuelas; es decir, privilegia el trabajo en torno de las estructuras aditivas, abordando más tarde el desarrollo de las estructuras de tipo multiplicativo, lo que dificulta el aprendizaje de la proporcionalidad

\section{Gráfico 2}
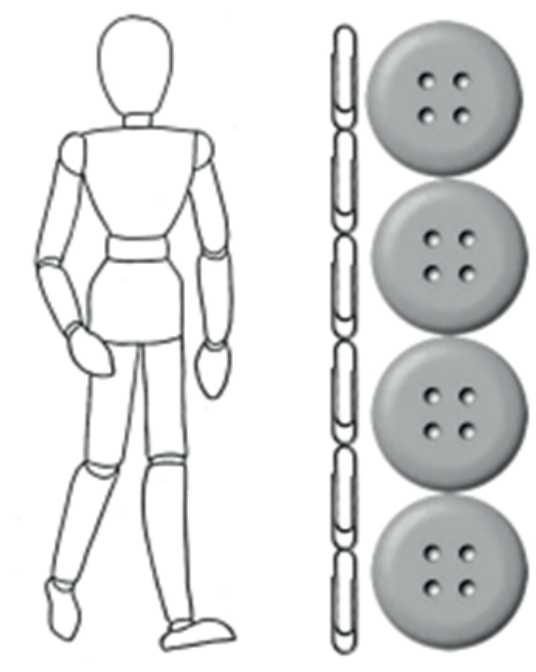

\section{Tú puedes ver la estatura} del Sr. Alto en "clips"

Tomado de Karplus y Peterson (1970)

Karplus y Peterson (1970) estableció como estrategias intuitivas las categorías I (Sin explicación), IC (usando la información, de una ma- nera ilógica), el 65\% de los niños usaron estos métodos y luego los habían sustituido. Una cantidad similar de niños pasó de la categoría A (usando toda la información, pero aplicando la diferencia, más que la razón de mediciones) a la S (usando la multiplicación, pero no a través del factor recto (usualmente mediante duplicación) porque no hubo base para ordenarlas. Estas categorías son relevantes porque a partir de ellas se pudo diversificar métodos para obtener las respuestas correctas.

En lo que refiere a las estrategias que utilizadas por los niños, Karplus, Pulos y Stage (1983) proponen cuatro categorías, producto del análisis de los diferentes métodos de solución empleados por los alumnos en tareas que involucran el razonamiento proporcional: eran los siguientes categorías: Incompleta: cuando adivinan la respuesta o emplean una operación cuantitativa inapropiada, Cualitativa, si comparan las cuatro cantidades dadas, usando los términos más, menos o términos equivalentes, Aditiva: al comparar los datos a partir de diferencias o residuos, Razonamiento proporcional, si usan datos para calcular y comparar relaciones proporcionales.

Los estudiantes fueron sometidos a las siguientes tareas: un problema (clips de papel, que consiste en buscar el valor ausente y tratar con razones fraccionarias. Noelting (1980) estudió el razonamiento proporcional con tareas que requerían de los sujetos la comparación de dos proporciones (jugo de naranja y agua, para preparar dos bebidas con el mismo o diferente sabor de naranja) con el requerimiento de que calcularan una respuesta que produjera la proporción deseada. Las cantidades totales de la mezcla ¿saben igual? Los resultados expresan las dificultades de los niños en los problemas de proporción en la covariación.

Por otro lado, Fernandez y Llinares (2011) analizaron algunas características del desarrollo del razonamiento proporcional en alumnos de primaria y secundaria a partir de la resolución de problemas proporcionales. Trabajaron con 755 estudiantes, les presentaron un cuestionario con doce problemas: cuatro problemas de proporcionalidad (dos con cantidades continuas y dos con cantidades discretas), cuatro problemas aditivos (dos con cantidades continuas y dos con cantidades discretas), y cuatro problemas distractores. Los resultados encontrados revelaron algunas estrategias de resolución: Respuestas proporcionales, respuestas aditivas y otras respuestas. Encontraron que el tipo de cantidad - discreta o continua- no influyó en las respuestas, mientras que el tipo de razón y relación si influyó. Lograron identificar cinco perfiles: 1 . Correcto proporcionan respuestas de acuerdo al tipo de problemas proporcionales; 2. Proporcional: respuestas proporcionales tanto a los problemas aditivos como a los proporcionales; 3 . Aditivo: dan respuestas aditivas a los dos tipos de problemas; 4 Dependiendo del tipo de razón: Los estudiantes dan respuestas proporcionales en los problemas con razones o relaciones multiplicativas enteras (I) y dan respuestas aditivas en los problemas con razones o relaciones multiplicativas no enteras (N); y 5. Otros: Los estudiantes emplean otras respuestas, consideradas incorrectas. Comentan que el perfil correcto aparece tardíamente, y conforme los alumnos avanzan de grado escolar el perfil aditivo disminuye y aumenta el proporcional, lo cual tiene relación con el currículo.

Sánchez (2013) analizó las ideas de razones y proporciones partir de un problema que implicó el reparto proporcional con alumnos de 11 a 14 años de edad. Los resultados revelaron que los estudiantes prefirieron recurrir al análisis cualitativo (en algunos casos el alumno utiliza la proporcionalidad directa, la razón como relator y en otros responde con base a su conocimiento social), mientras que el análisis cuantitativo, un menor porcentaje de alumnos recurrió a ese tipo de análisis y su respuestas fueron básicamente estrategias aditivas. 
Obando, Vasco y Arboleda (2014), realizaron una revisión de la literatura de los últimos cincuenta años sobre el tema de razones, proporciones y proporcionalidad. Estos autores comentan que, de acuerdo a Karplus et al., (1983), los estudiantes presentan dificultades en lo referente a su aprendizaje; y mencionan que existen problemas en la enseñanza de ese contenido escolar. Concluyen que es un tema que requiere más investigaciones de corte didáctico, que permitan conocer la problemática que presentan los alumnos para poder tener un cierto nivel de impacto en el proceso de enseñanza y aprendizaje de ese contenido escolar.

Por otro lado, de acuerdo con los planes y programas de estudio de la Secretaria de Educación Pública (SEP 2011, 2016), el estudio de la proporcionalidad se lleva a cabo en los dos últimos grados de educación primaria y primeros grados de la educación secundaria. Éste constituye un eje articulador de contenidos matemáticos diversos, cuyo estudio continúa y se generaliza en los niveles posteriores. Este contenido escolar también está presente en las relaciones multiplicativas y está fuertemente relacionado a las nociones de multiplicación, división, número racional, escala, porcentaje y probabilidad, entre otras ideas matemáticas.

A manera de síntesis podemos decir, que el estudio del razonamiento proporcional se lleva a cabo en los dos últimos grados de educación primaria y primeros grados de la educación secundaria. Éste constituye un eje articulador de contenidos matemáticos diversos, cuyo estudio continúa y se generaliza en los niveles posteriores. En la educación secundaria, el razonamiento proporcional se estudia con las herramientas del álgebra como funciones lineales: se pone énfasis en obtener su expresión algebraica y sus gráficas, así como también en el análisis de sus características estructurales. Este paso del estudio de la proporcionalidad al estudio de las funciones lineales corresponde a un doble tránsito: de un campo de conocimiento aritmético a un algebraico, y entre diferentes niveles de estudios, de la educación primaria a la secundaria y de la educación secundaria a la educación media superior.

Estos antecedentes orientan este estudio como una manera para validar los constructos del razonamiento proporcional como una vía para acceder al pensamiento algebraico temprano con estudiantes de los últimos grados de educación primaria ( $5^{\circ}$ y 60 grado) y ler grado de educación secundaria, como también busca identificar el desempeño en relación al grado escolar por medio de mediciones sobre el razonamiento proporcional.

\section{Metodología}

Estudio de tipo observacional, longitudinal y comparativo. Se estudiaron a tres grupos de estudiantes a lo largo de un año.

\section{Participantes}

Para la evaluación del razonamiento proporcional, fueron evaluados 109 estudiantes del Estado de México; y de la Ciudad de México que cursaban 4 to, $5^{\circ}$ grados y ler grado de secundaria.

Es importante mencionar que los estudiantes eran originarios de un nivel socio-económico medio. El criterio de inclusión para el estudio fue que los estudiantes hubieran concluido el ciclo escolar.

\section{Elaboración de instrumento}

Para la elaboración del instrumento de evaluación para el razonamiento proporcional se utilizó el Programas de estudios de la Secretaria de Educación Púbica de quinto y sexto grado de primaria (SEP, 2011.2016), y el libro de texto gratuito de matemáticas.

Se realizó un análisis documental de los planes y programas de estudio de la Secretaría de Educación Pública de $5^{\circ}$ y 60 grados de educación primaria. Con base en ese análisis se realizó una descripción de los aprendizajes esperados y se reestructuró el instrumento de evaluación.

Con base en estos análisis y considerando la organización curricular de la enseñanza de matemáticas en quinto y sexto grado de educación primaria, se eligieron los aprendizajes esperados más representativos del razonamiento proporcional con el objetivo de rastrear los aprendizajes desarrollados en dichos grados escolares. Fueron elegidos los aprendizajes esperados por sus características estructurales y que también pudieran ser evaluados con preguntas en papel y lápiz.

\section{Diseño y descripción del instrumento de razonamiento proporcional}

Con apego a los programas de estudio de matemáticas para el nivel básico, se elaboró un cuestionario que exploró ideas básicas sobre el razonamiento proporcional. Esta versión fue presentada a dos especialistas en enseñanza de las matemáticas; y ello sirvió para modificar algunas preguntas y complementar otras. Cabe mencionar que, de acuerdo con el Plan y Programas de estudios de la Secretaria de Educación Básica (SEP), éste incluye el razonamiento proporcional.

El proceso de construcción del cuestionario sobre razonamiento proporcional consistió en delimitar las competencias que los estudiantes debían mostrar sobre este contenido escolar explorado en el instrumento. Los indicadores de ejecución y los conocimientos sobre el razonamiento proporcional con las seis dimensiones y sus respectivos indicadores: Invarianza de la proporción, Proporción geométrica, Proporción aritmética, Relación Lineal, Proporción y escala, Proporción entre varias cantidades.

\section{Validación del instrumento sobre razonamiento proporcional}

El instrumento para evaluar el razonamiento proporcional fue construido desde las seis dimensiones sobre razonamiento proporcional, este instrumento fue sometido a una valoración de contenido por dos expertos sobre la enseñanza de las matemáticas y fue útil para hacer ajustes al instrumento.

Posteriormente el instrumento fue aplicado a una muestra piloto conformada por 50 estudiantes de 4050 y 60 grados de primaria y 50 estudiantes de primero de secundaria. Esos estudiantes eran diferentes a los estudiantes que conformaron el estudio principal. 
A partir de las observaciones de los expertos y los resultados de la prueba piloto, se rediseño el instrumento y al término del ciclo escolar, tres evaluadores aplicaron de manera colectiva, la versión final del instrumento a 109 niños, en tres sesiones de 40 minutos de duración cada una.

Tabla 1 Descripción del instrumento para evaluar el razonamiento proporcional
En la tabla 1 se describe la estructura del instrumento utilizado en el estudio, éste incluye las cuatro dimensiones (factores), las descripciones, los indicadores y el contenido matemático evaluado.

\begin{tabular}{|c|c|c|c|}
\hline $\begin{array}{l}\text { Dominios Razonamiento } \\
\text { Proporcional }\end{array}$ & $\begin{array}{l}\text { Descripción y clave de la dimensión } \\
\text { (variable) }\end{array}$ & Indicadores & Contenido matemático \\
\hline \multirow[t]{3}{*}{$\begin{array}{l}\text { 1. Invariancia de Proporción } \\
\text { (Reconocer la invariancia de la } \\
\text { proporción ante movimientos } \\
\text { rígidos) }\end{array}$} & $\begin{array}{l}\text { Reconocer la invariancia de la } \\
\text { proporción ante movimientos rígidos } \\
\text { e incluye tres subdominios. }\end{array}$ & $\begin{array}{l}\text { InvaProA. } \\
\text { Invariancia de la proporción, } \\
\text { elección de la figura. }\end{array}$ & $\begin{array}{l}\text { Reconocer la invariancia de la } \\
\text { proporción ante movimientos } \\
\text { rígidos, elección de la figura. }\end{array}$ \\
\hline & & $\begin{array}{l}\text { InvaProB. } \\
\text { Invariancia de la proporción, } \\
\text { argumento }\end{array}$ & $\begin{array}{l}\text { Reconocer la invariancia de la } \\
\text { proporción ante movimientos } \\
\text { rígidos, argumento (posición) }\end{array}$ \\
\hline & & $\begin{array}{l}\text { Invariancia de la proporción, } \\
\text { diferencia del resto de figuras }\end{array}$ & $\begin{array}{l}\text { Reconocer la invariancia de la } \\
\text { proporción ante movimientos } \\
\text { rígidos, diferencias del resto de las } \\
\text { figuras. }\end{array}$ \\
\hline 2. Proporción Geométrica. & $\begin{array}{l}\text { Reconocer una proporción } \\
\text { geométrica en una secuencia de } \\
\text { rectángulos, incluye cuatro } \\
\text { subdominios. }\end{array}$ & Propor1completar & $\begin{array}{l}\text { Reconocimiento de una proporción } \\
\text { geométrica en una secuencia de } \\
\text { rectángulos (9) }\end{array}$ \\
\hline
\end{tabular}

Propor2 completar

Reconocimiento de una proporción geométrica en una secuencia de rectángulos (3)

Propor3 completar

Reconocimiento de una proporción geométrica en una secuencia de rectángulos (18)

Reconocimiento de una proporción

Propor4 completar geométrica en una secuencia de rectángulos (20)

\begin{tabular}{lll}
\hline 3. Proporción Aritmética $\quad$ Proporción aritmética & Proporcionalidad aritmética & $\begin{array}{l}\text { Proporcionalidad aritmética entre } \\
\text { litros de agua para } 8 \text { personas }\end{array}$
\end{tabular}

Proporcionalidad aritmética entre litros de agua para 4 personas

\begin{tabular}{|c|c|c|}
\hline & & $\begin{array}{l}\text { Proporcionalidad aritmética entre } \\
\text { litros de agua para } 32 \text { personas }\end{array}$ \\
\hline & & $\begin{array}{l}\text { Proporcionalidad aritmética, tabla } \\
\text { de datos }\end{array}$ \\
\hline & & Relación aritmética lineal (limonada) \\
\hline \multirow[t]{3}{*}{ 4. Relación Lineal } & $\begin{array}{l}\text { Descripción Compuesta por tres } \\
\text { subdominios. }\end{array}$ & Proporción geométrica, gráfica \\
\hline & & $\begin{array}{l}\text { Relación lineal proporción de litros } \\
\text { de agua por persona }\end{array}$ \\
\hline & & $\begin{array}{l}\text { Relación lineal elaboración de una } \\
\text { regla }\end{array}$ \\
\hline \multirow[t]{5}{*}{ 5. Proporción y Escala } & $\begin{array}{l}\text { Descripción Formada por cinco } \\
\text { subdominios. }\end{array}$ & Proporción y escala de 1 a 3 (Casita) \\
\hline & & $\begin{array}{l}\text { Mantener proporción dada escala } 1 \text { a } \\
3 \text { (carrito) }\end{array}$ \\
\hline & & $\begin{array}{l}\text { Mantener la proporción ¿Cómo lo } \\
\text { obtuviste? }\end{array}$ \\
\hline & & $\begin{array}{l}\text { Mantener la proporción ¿Cuántas } \\
\text { veces se amplió? }\end{array}$ \\
\hline & & $\begin{array}{l}\text { Mantener la proporción, elaborar una } \\
\text { regla }\end{array}$ \\
\hline 6. Proporción entre varias cantidades & $\begin{array}{l}\text { Descripción Compuesta por cuatro } \\
\text { subdominios. }\end{array}$ & $\begin{array}{l}\text { Proporción entre varias cantidades } \\
\text { (parejas de rectángulos) }\end{array}$ \\
\hline
\end{tabular}




\section{Entrevista clínica individual}

Posteriormente, se aplicó una entrevista clínica piagetiana individual. De acuerdo a Delval (2011) la entrevista clínica es un método para investigar cómo piensan, perciben, actúan y sienten los estudiantes. La entrevista tuvo como objetivo indagar sobre cómo resolvieron el cuestionario los estudiantes, las estrategias que utilizaban para responder al cuestionario, así como también sirvió para indagar sobre las habilidades y dificultades que los estudiantes presentaban al responder al instrumento.

\section{Niveles de logro para el cuestionario de razonamiento proporcional}

Las respuestas de los estudiantes al cuestionario sobre razonamiento proporcional se analizaron de acuerdo a dos grandes categorías: niveles de logro académico y estrategias de resolución de problemas. Los niveles de logro surgen de las respuestas de los estudiantes al cuestionario inicial sobre razonamiento proporcional y estos son vistos como parte del desarrollo del pensamiento de los niños. Estos niveles de logro no son estáticos ni fijos y cambian a medida que los niños avanzan en la instrucción escolar.

Se elaboraron tres niveles de logro a partir de las respuestas que los estudiantes daban en el cuestionario inicial. A continuación, se describen:
Niveles de logro: los niveles de logro se entienden como una especie de ruta de proceso del estudiante en lo que respecta al tipo de pregunta, contenido matemático que presentan los estudiantes en una determinada tarea (SIMCE, 2007); pueden servir para guiar al docente sobre las necesidades educativas de los alumnos; fueron elaborados a partir de las respuestas de los alumnos al cuestionario inicial de razonamiento proporcional. A continuación, se describen los niveles de logro encontrados: nivel de logro inicial, medio y alto.

Nivel de logro inicial: Los alumnos que se encuentran en este nivel emplean la percepción intuitiva y la observación. Utilizan un pensamiento predominantemente aditivo.

Nivel de logro medio: Los alumnos que se encuentran en este nivel realizan las tareas usando una estrategia aditiva o en ocasiones un pensamiento multiplicativo incompleto.

Nivel de logro alto: Los alumnos que se encuentran en este nivel realizan las tareas usando una estrategia multiplicativa y pueden encontrar una regla que expresa la proporción algebraica.

\section{Estrategias de resolución de problemas}

A continuación, se muestran las categorías de resolución de problemas encontradas en el instrumento sobre razonamiento proporcional.

Tabla 2 Descripción de las estrategias de resolución de problemas del cuestionario de razonamiento proporcional.

\begin{tabular}{|c|c|}
\hline $\begin{array}{l}\text { Categorias de resolución de } \\
\text { problemas }\end{array}$ & Descripción de las categorías de resolución de problemas \\
\hline $\begin{array}{l}\text { Percepción intuitiva de la } \\
\text { proporcionalidad }\end{array}$ & $\begin{array}{l}\text { En esta categoría el estudiante realiza una comparación } \\
\text { cualitativa para identificar y reproducir objetos semejantes. }\end{array}$ \\
\hline Pensamiento en transición & $\begin{array}{l}\text { En esta categoría el estudiante usa su pensamiento intuitivo } \\
\text { y empieza a tomar en cuenta la información numérica, aún } \\
\text { no aplica la misma estrategia aditiva durante todo el } \\
\text { proceso de resolución. }\end{array}$ \\
\hline Pensamiento aditivo & $\begin{array}{l}\text { En esta categoría el estudiante resuelve el problema } \\
\text { utilizando una estrategia aditiva. }\end{array}$ \\
\hline $\begin{array}{l}\text { Pensamiento multiplicativo } \\
\text { incompleto }\end{array}$ & $\begin{array}{l}\text { En esta categoría el estudiante resuelve la tarea usando la } \\
\text { multiplicación, aún no aplica la misma estrategia aditiva } \\
\text { durante todo el proceso de resolución y no considera la } \\
\text { relación de todos los datos del problema. }\end{array}$ \\
\hline $\begin{array}{l}\text { Pensamiento multiplicativo } \\
\text { completo }\end{array}$ & $\begin{array}{l}\text { En esta categoría el estudiante percibe la percepción } \\
\text { geométrica, considera la información numérica, relaciona } \\
\text { todos los datos o variables y resuelve el problema haciendo } \\
\text { uso de la multiplicación. }\end{array}$ \\
\hline
\end{tabular}

\section{Resultados}

Consistencia interna en la medición del cuestionario sobre razonamiento proporcional

Los resultados sobre la consistencia interna, se obtuvo un coeficiente general de Alpha de Cronbach de 0.83, esto significa que hay una buena consistencia interna del instrumento para medir los indi- cadores sobre razonamiento proporcional con estas tareas en sus seis distintas dimensiones. Además, la consistencia interna por dimensión es la siguiente, para Invariancia de la proporción se obtuvo un alpha de 0.74; Proporción Geométrica 0.83; Proporcionalidad aritmética 0.85; algebraica 0.24 y finalmente para la dimensión de Escala 1 a 3 se obtuvo un alpha de 0.73 


\section{Validez de constructo para el razonamiento proporcional}

Paraelanálisisfactorialconfirmatorioseutilizó el programaEQS6(Bentler \& Wu. 2002), con el objetivo de verificar las preguntas o indicadores de acuerdo con los seis dominios de razonamiento proporcional y además fueron estructurados con base en investigaciones sobre este contenido escolar. Para tal objetivo, se organizó un modelo de análisis factorial confirmatorio (figura 3), con el objetivo de obtener la validez convergente y divergente de constructo. Se indica con círculos las cuatro dimensiones (constructos), y con rectángulos amarillos se exponen los indicadores o variables manifiestas de cada dimensión del razonamiento proporcional.

Gráfico 3 Modelo hipotético de validez convergente y divergente de constructo en la medición del razonamiento proporcional.: Análisis Factorial Confirmatorio.

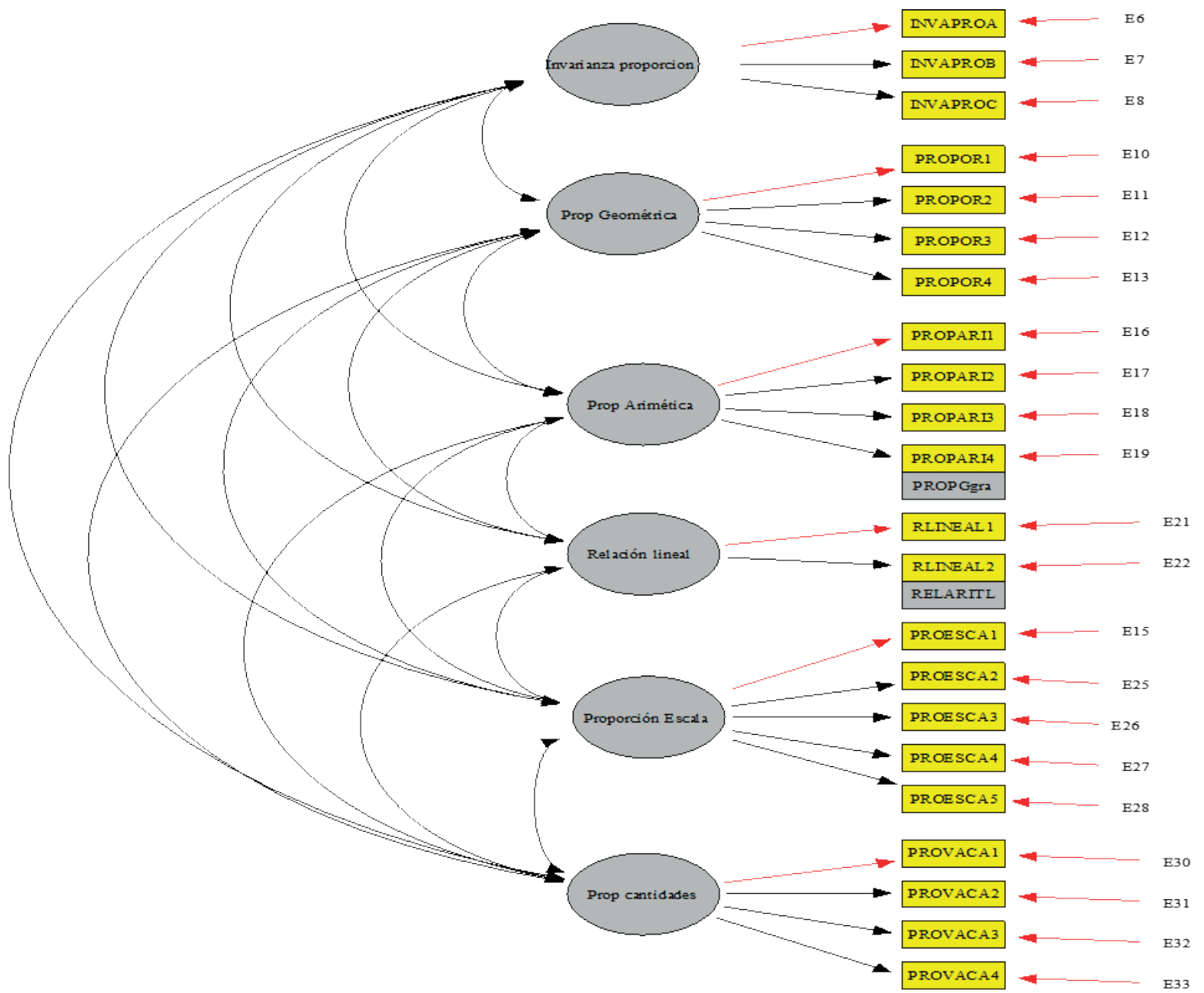

Gráfico 4 Modelo resultante de validez convergente y divergente de constructo en la medición de razonamiento proporcional: Análisis factorial confirmatorio

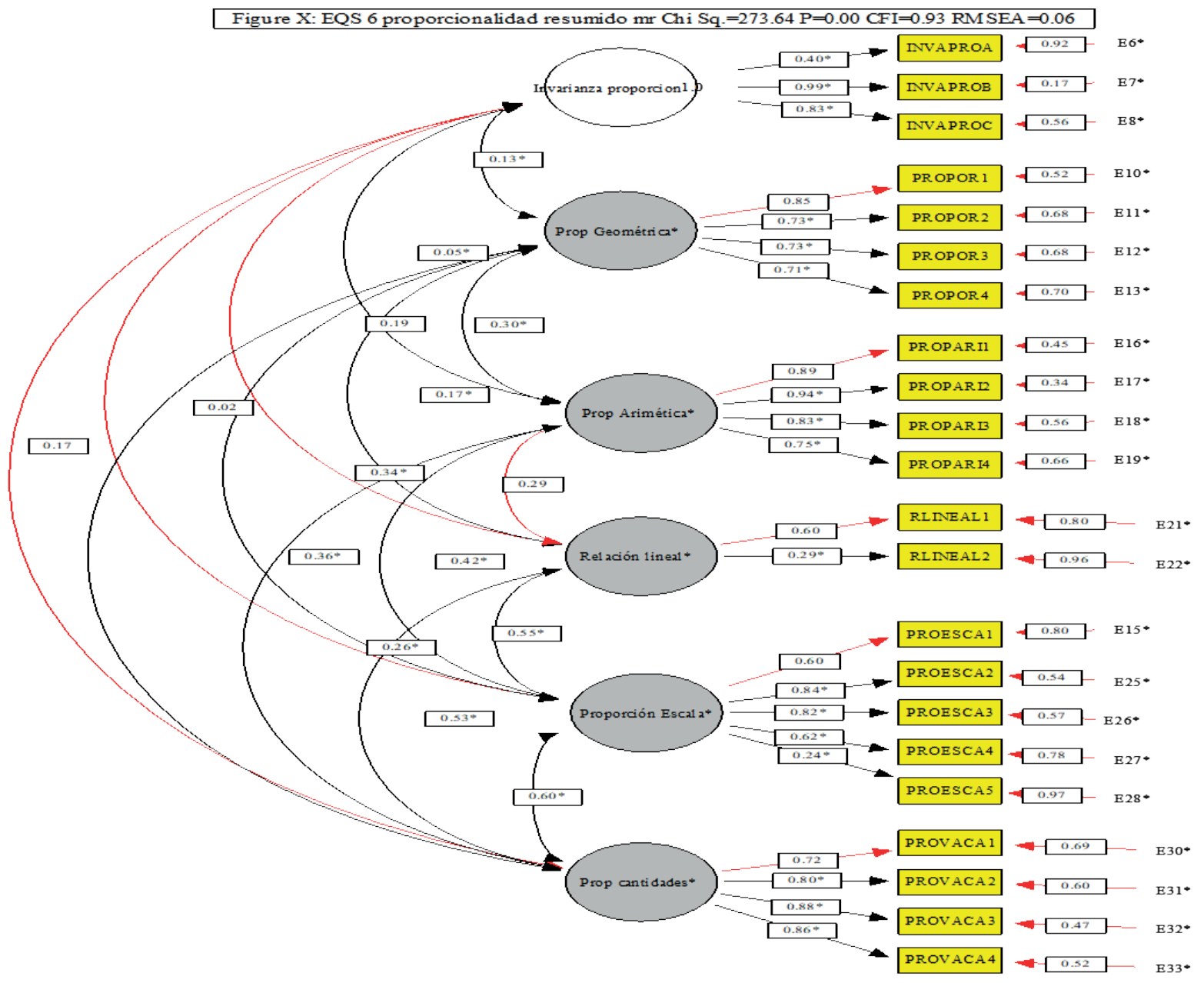




\section{El razonamiento proporcional en educación básica}

El modelo supone que hay validez convergente cuando se obtienen pesos factoriales significativos y similares entre cada constructo (indicado en círculo) y sus indicadores (representados en rectse muestra la tabla... estudio uy cirricularmente.res, como los procesos de generalizacincativossiento prporcionaliños, en dos sángulos) Las flechas de un sentido (de los círculos a los rectángulos) indican que estas variables son indicadores de ese constructo o factor. Las flechas direccionales entre círculos indican que un factor o variable latente influye en otro factor o variables latente. La Letra E indica el error asociado con la medida o características que el instrumento no está recogiendo en relación con los indicadores o variables manifiestas, o cuestiones del azar (Bazán Ramírez, Sánchez Hernández, \& Castañeda Figueiras, 2007; Bazán Ramírez, Sánchez Hernández, Corral Verdugo, \& Castañeda Figueiras, 2006; Byrne, 1994).

El modelo resultante de AFC (Gráfico 4), permite observar la configuración de un modelo con seis dimensiones de razonamiento proporcional con todos los indicadores incluidos en el modelo hipotético. El modelo resultante obtuvo aceptables indicadores prácticos de bondad de ajuste ( $P=0.00, N F I=0.90 ; C F|=0.92 ;| F \mid=0.92 ; R M S E A=0.07), y$ coeficientes de confiabilidad altos ( $\alpha=0.84 ; \mathrm{RHO}=0.87$ ).

\section{Resultados datos comparativos por Estado}

En lo que refiere a los datos comparativos por estado (entidad federativa): podemos observar que el lugar en donde estudian (estado) no es influyente para el razonamiento proporcional. Pues la desviación media es casi igual en todas las dimensiones que se evaluaron, pues la media para el Promedio de Varianza de la proporción en el DF es de 0.9487, y para el Estado de México 0.986; en el Promedio de Proporción geométrica se obtuvo para el DF 1.4063, y para el Estado de México 1.7552; posteriormente el Promedio de Proporción aritmética en el DF es de 1.4152 y para el Estado de México es de 1.3333, finalmente en el Promedio Relación lineal se obtuvo para el DF una media de 1.3631, y para el Estado de México 1.2917.

\section{Resultados: niveles de logro}

A continuación, se muestra un cuadro de los datos encontrados por nivel de logro de los alumnos de educación primaria que participan en el estudio y que cursan el $5^{\circ}$ grado de primaria.

Tabla 3 Frecuencias de Respuesta y Porcentajes Razonamiento Proporcional

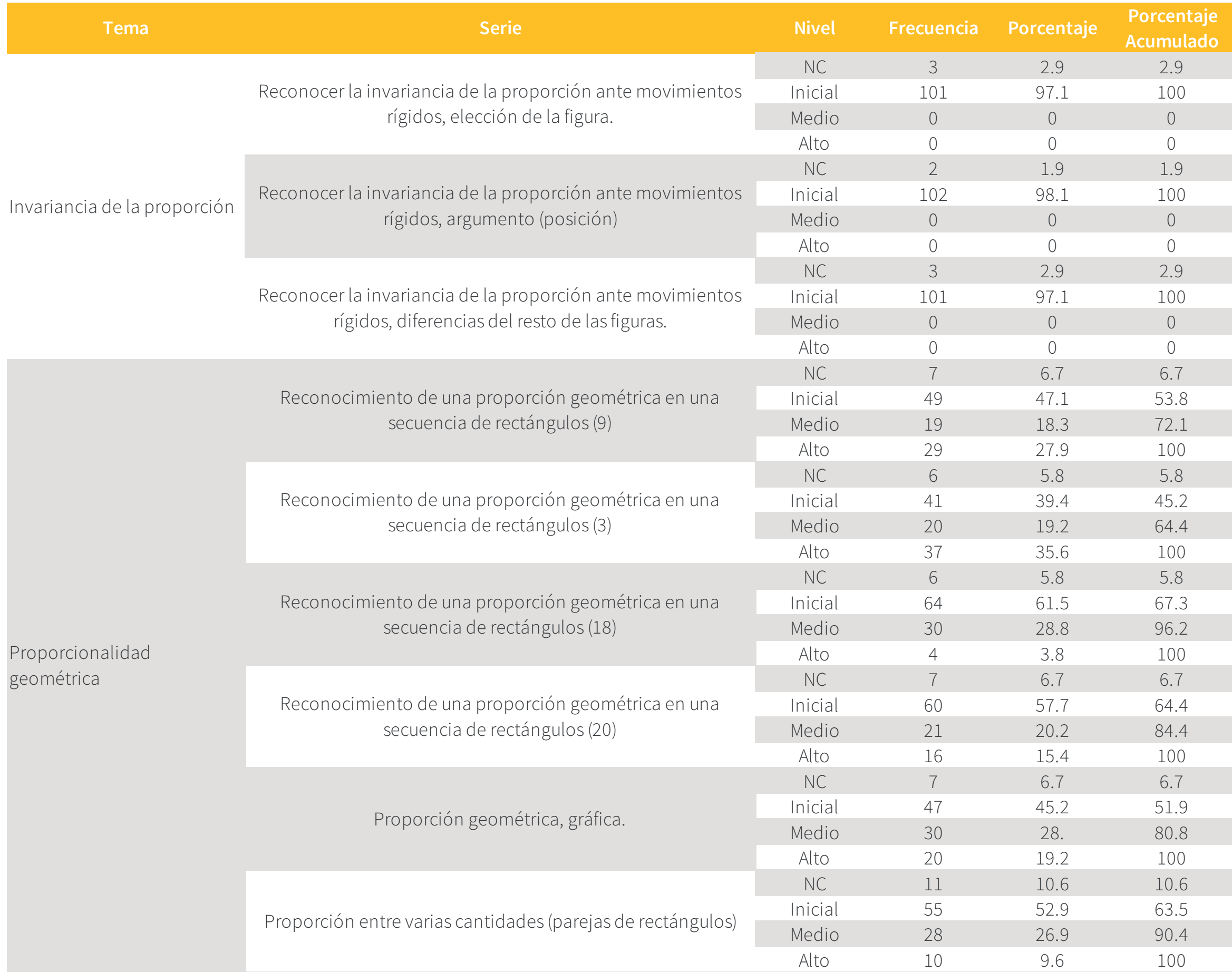




\begin{tabular}{|c|c|c|c|c|c|}
\hline Tema & Serie & Nivel & Frecuencia & Porcentaje & $\begin{array}{l}\text { Porcentaje } \\
\text { Acumulado }\end{array}$ \\
\hline \multirow{12}{*}{$\begin{array}{l}\text { Proporcionalidad } \\
\text { geométrica }\end{array}$} & \multirow{4}{*}{ Proporción entre varias cantidades ¿qué tomaste en cuenta? } & NC & 4 & 3.8 & 3.8 \\
\hline & & Inicial & 45 & 43.3 & 47.1 \\
\hline & & Medio & 41 & 39.4 & 86.5 \\
\hline & & Alto & 14 & 13.5 & 100 \\
\hline & \multirow{4}{*}{ Proporción entre varias cantidades, tabla } & NC & 14 & 13.5 & 13.5 \\
\hline & & Inicial & 53 & 51 & 64.4 \\
\hline & & Medio & 29 & 27.9 & 92.3 \\
\hline & & Alto & 8 & 7.7 & 100 \\
\hline & \multirow{4}{*}{$\begin{array}{l}\text { Proporción entre varias cantidades, ¿por qué son } \\
\text { proporcionales? }\end{array}$} & NC & 22 & 21.2 & 21.2 \\
\hline & & Inicial & 46 & 44.2 & 65.4 \\
\hline & & Medio & 26 & 25.0 & 90.4 \\
\hline & & Alto & 10 & 9.6 & 100 \\
\hline \multirow{20}{*}{ Proporcionalidad aritmética } & \multirow{4}{*}{$\begin{array}{l}\text { Proporcionalidad aritmética entre litros de agua para } 8 \\
\text { personas }\end{array}$} & NC & 14 & 13.5 & 13.5 \\
\hline & & Inicial & 0 & 0 & 13.5 \\
\hline & & Medio & 25 & 24.0 & 37.5 \\
\hline & & Alto & 65 & 62.5 & 100 \\
\hline & \multirow{4}{*}{$\begin{array}{l}\text { Proporcionalidad aritmética entre litros de agua para } 4 \\
\text { personas }\end{array}$} & NC & 1 & 1.0 & 1.0 \\
\hline & & Inicial & 16 & 15.4 & 16.3 \\
\hline & & Medio & 26 & 25.0 & 41.3 \\
\hline & & Alto & 61 & 58.7 & 100 \\
\hline & \multirow{4}{*}{$\begin{array}{c}\text { Proporcionalidad aritmética entre litros de agua para } 32 \\
\text { personas }\end{array}$} & NC & 1 & 1.0 & 1.0 \\
\hline & & Inicial & 27 & 26.0 & 26.9 \\
\hline & & Medio & 18 & 17.3 & 44.2 \\
\hline & & Alto & 58 & 55.8 & 100 \\
\hline & \multirow{4}{*}{ Proporcionalidad aritmética, tabla de datos } & NC & 20 & 19.2 & 19.2 \\
\hline & & Inicial & 0 & 0 & 19.2 \\
\hline & & Medio & 31 & 29.8 & 49.0 \\
\hline & & Alto & 53 & 51 & 100 \\
\hline & \multirow{4}{*}{ Relación aritmética lineal (limonada) } & NC & 34 & 32.7 & 32.7 \\
\hline & & Inicial & 0 & 0 & 32.7 \\
\hline & & Medio & 13 & 12.5 & 45.2 \\
\hline & & Alto & 57 & 54.8 & 100 \\
\hline \multirow{8}{*}{ Algebraica } & \multirow{4}{*}{ Proporción algebraica entre litros de agua por persona, } & NC & 9 & 8.7 & 8.7 \\
\hline & & Inicial & 63 & 60.6 & 69.2 \\
\hline & & Medio & 15 & 14.4 & 83.7 \\
\hline & & Alto & 17 & 16.3 & 100 \\
\hline & \multirow{4}{*}{ Proporción algebraica, elaboración de una regla } & $N C$ & 11 & 10.6 & 10.9 \\
\hline & & Inicial & 83 & 79.8 & 90.4 \\
\hline & & Medio & 9 & 8.7 & 99.0 \\
\hline & & Alto & 1 & 1.0 & 100 \\
\hline \multirow{20}{*}{ Escala 1 a 3} & \multirow{4}{*}{ Proporción y escala de 1 a 3 (Casita) } & NC & 1 & 1.0 & 1.0 \\
\hline & & Inicial & 47 & 45.2 & 46.2 \\
\hline & & Medio & 39 & 37.5 & 83.7 \\
\hline & & Alto & 17 & 16.3 & 100 \\
\hline & \multirow{4}{*}{ Mantener proporción dada escala 1 a 3 (carrito) } & $N C$ & 4 & 3.8 & 3.8 \\
\hline & & Inicial & 37 & 35.6 & 39.4 \\
\hline & & Medio & 18 & 17.3 & 56.7 \\
\hline & & Alto & 45 & 43.3 & 100 \\
\hline & & $N C$ & 3 & 2.9 & 2.9 \\
\hline & Mantener la proporción icómo lo obtuviste? & Inicial & 30 & 28.8 & 31.7 \\
\hline & mianlenter la proporion zcomo lo oncuviste? & Medio & 41 & 39.4 & 71.2 \\
\hline & & Alto & 30 & 28.8 & 100 \\
\hline & & NC & 6 & 5.8 & 5.8 \\
\hline & Mantener la pronorción icuántas veces se amnlió? & Inicial & 19 & 18.3 & 24.0 \\
\hline & Mancener la proporcion ¿cuanlas veces se ampilo? & Medio & 22 & 21.2 & 45.2 \\
\hline & & Alto & 57 & 54.8 & 100 \\
\hline & & NC & 49 & 47.1 & 47.1 \\
\hline & Mantener la proporción, elaborar una regla & Inicial & 43 & 41.3 & 88.5 \\
\hline & Mancener la proporcion, elaborar una regia & Medio & 8 & 7.7 & 96.2 \\
\hline & & Alto & 4 & 3.8 & 100 \\
\hline
\end{tabular}




\section{El razonamiento proporcional en educación básica}

La Tabla anterior nos muestra las frecuencias de respuesta de los alumnos quienes se aplicó el instrumento, en cuatro niveles de ejecución (Inicial, Medio, Alto) y un No Contesta. Dentro de la primera dimensión (Invarianza de la proporción) los tres indicadores en su gran mayoría puntúan en el nivel inicial. Lo mismo pasa con la dimensión Proporción geométrica y la dimensión Algebraica; todos sus indicadores se quedan con respuestas de nivel de ejecución inicial.

Por otra parte, la dimensión de Proporcionalidad aritmética sube al nivel de ejecución alto en todos sus indicadores, y finalmente, en la última dimensión (Escala 1 a 3), los niveles de ejecución se quedan el inicial en dos de sus indicadores (1 y 4) y en los otros dos (2 y 3 ) en el nivel alto. Este cuadro permite establecer parámetros para definir el análisis de los resultados. Por ejemplo, en la pregunta en donde se explora la percepción intuitiva, sólo es posible identificar estrategias de un pensamiento intuitivo; es decir, la pregunta no ofrece información numérica que pueda ser tratada de manera aditiva o multiplicativa, sino demanda de los estudiantes reconocer los criterios cualitativos para comparar y establecer la relación de proporción ante movimientos rígidos de la figura. Sin embargo, en las respuestas de los estudiantes hay evidencias del uso de estrategias de resolución de problemas en la categoría del pensamiento intuitivo, en la totalidad de las preguntas; sobre todo, se observa cuando se explora la proporcionalidad geométrica.

También es posible observar que, considerando que el nivel de logro alto representaría el desarrollo del conocimiento algebraico, se caracterizan las respuestas de los estudiantes cuando usan estrategias multiplicativas completas en donde identifican el patrón y pueden o no elaborar una regla utilizando un lenguaje matemático. Esta condi- ción sólo se observa en las preguntas 4 y 6 , en donde se les solicita a los estudiantes operar con la información a fin de identificar la proporcionalidad y expresarla mediante la elaboración de una regla

\section{Análisis cualitativo}

El estudio también tuvo una fase de análisis cualitativo. El objetivo de dicho análisis consistió en categorizar las respuestas de los estudiantes para el cuestionario de razonamiento proporcional. Se encontraron una gran variedad de respuestas de los alumnos.

Estrategias de resolución de problemas para el cuestionario de razonamiento proporcional

A partir de las respuestas de los estudiantes al cuestionario de razonamiento proporcional, se elaboraron categorías de análisis de acuerdo a las respuestas de los estudiantes y las denominamos estrategias de resolución de problemas como las que se muestran en la tabla número 4. Estas estrategias indican cómo los estudiantes resolvían las preguntas planteadas en el cuestionario de proporcionalidad, además estas respuestas también revelan el nivel de entendimiento conceptual del contenido escolar explorado en el instrumento.

A continuación, en la tabla 4, se muestran las estrategias de resolución de problemas encontradas. Se indican inicialmente las categorías de resolución de problemas, luego se presenta un ejemplo de la respuesta de los alumnos para dicha categoría y se presenta un breve comentario sobre esa categoría de resolución de problema.

Tabla 4 Resultados Estrategias de resolución de problemas para el cuestionario de razonamiento proporcional.
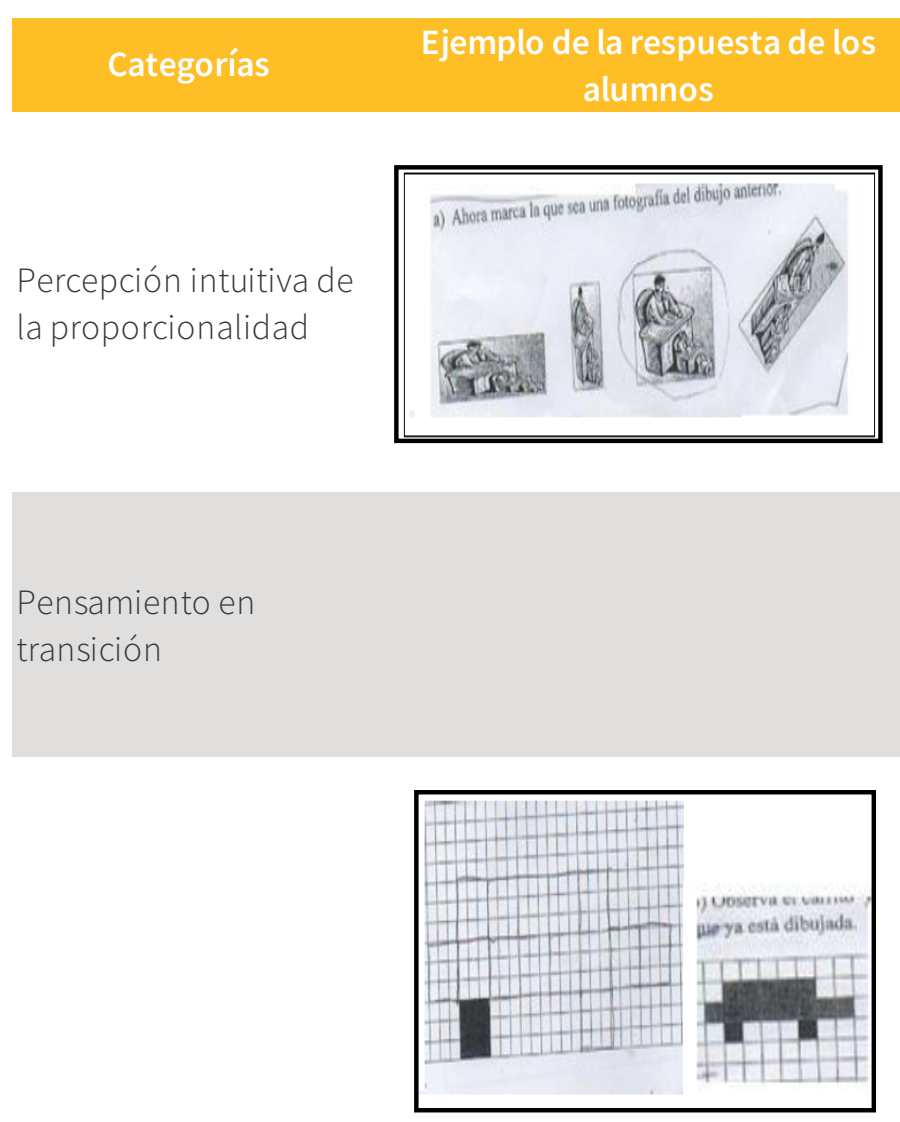

12
Pensamiento aditivo

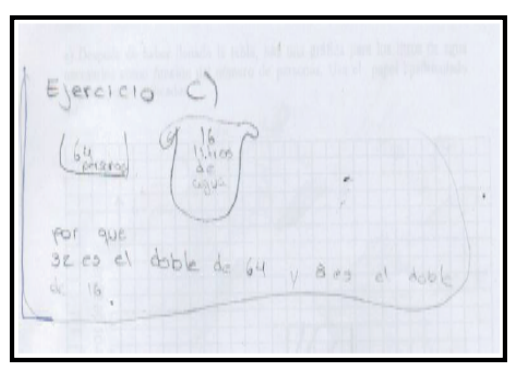

En esta categoría los alumnos emplean un pensamiento intuitivo; mediante la descripción de características o cualidades físicas del objeto encuentran el elemento correspondiente al original y luego identifican una de las fotografías. Ellos empiezan a reconocer propiedades como la forma expandida, chueca, borrosa, volteada, alargada; el contorno, si es largo o angosto; grande o chico.

En esta categoría los alumnos usan un pensamiento en transición muestran todavía dificultad para establecer las relaciones de proporción entre las cantidades; por ejemplo, cuando hay que buscar la medida faltante de una serie de rectángulos.

En esta categoría los alumnos usan un pensamiento intuitivo y empiezan a tomar en cuenta la información numérica, pero aún no aplican la misma estrategia aditiva durante todo el proceso de resolución. Los alumnos tratan de responder a preguntas donde hay que realizar cálculos a partir de la suma, sin embargo, se les dificulta alcanzar el resultado correcto. En esta categoría los alumnos de quinto emplean la estrategia aditiva cuando resuelve problemas de proporcionalidad. Por ejemplo, emplean la suma como estrategia en las tareas No. 2 (serie de rectángulos), No. 3 (dibujo de casita a escala), No. 5 (limonada) y No. 6 (dibujo a escala carrito); esta estrategia se les facilita cuando realizan comparaciones cuantitativas; sin embargo, esta no necesariamente es la vía para encontrar la respuesta correcta; es decir, las tareas se pueden resolver por medio de cálculos de suma, pero, estas tareas están diseñadas para que el alumno utilice la adición y en mayor medida la multiplicación. 


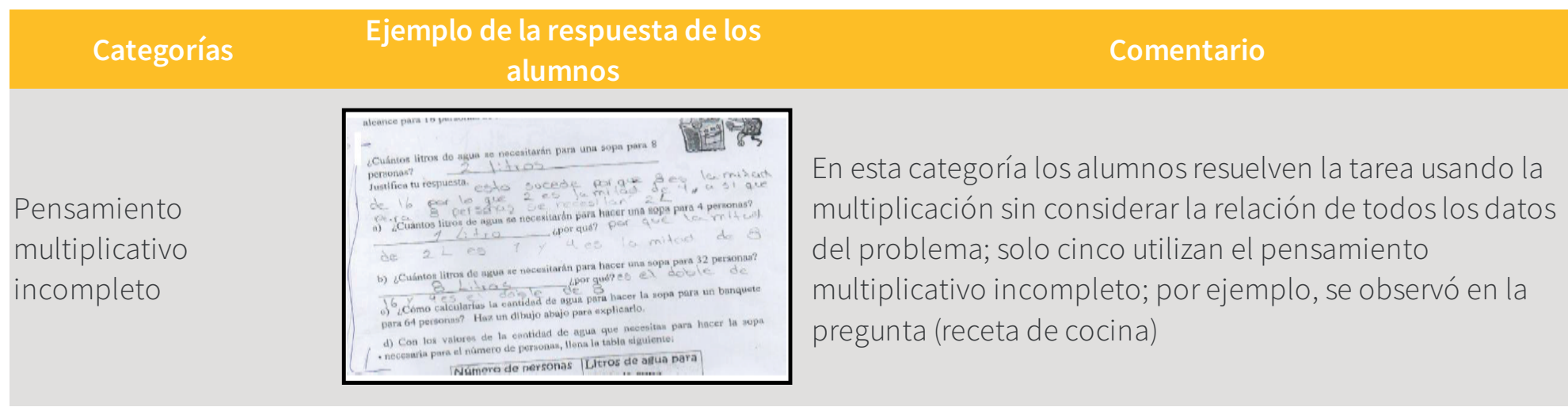

Pensamiento

multiplicativo

completo

En esta categoría los alumnos alertan la percepción geométrica, consideran la información numérica, relacionan todos los datos o variables y resuelven el problema usando la multiplicación; esto se pudo observar en las preguntas (casa a escala) se espera que la mayoría logren este pensamiento al terminar la educación básica.

\section{Conclusiones}

Los resultados de este estudio revelan, por un lado, la existencia de habilidades, pero también dificultades típicas de esas edades (transición del pensamiento aditivo al pensamiento multiplicativo), en parte por el énfasis que la instrucción escolar imprime en los problemas de estructura aditiva y deja de lado el desarrollo del pensamiento multiplicativo que propicia, entre otras cosas, el desarrollo de un pensamiento más complejo y abstracto por medio de la resolución de problemas de estructura multiplicativa.

En lo que refiere a los resultados del estudio, específicamente al instrumento para evaluar el razonamiento proporcional fue construido desde las seis dimensiones sobre razonamiento proporcional, este instrumento fue sometido a una valoración de contenido por dos expertos sobre la enseñanza de las matemáticas y fue útil para hacer ajustes al instrumento.

Para validar el instrumento, se realizó una validez de constructo para el cuestionario de razonamiento proporcional, se propuso un modelo hipotético de validez convergente y divergente de constructo en la medición del razonamiento proporcional y el modelo resultante obtuvo aceptables indicadores prácticos de bondad de ajuste y coeficientes de confiabilidad altos.

A partir de los resultados sobre consistencia interna, éstos revelaron que hay una buena consistencia interna del instrumento para medir los indicadores sobre razonamiento proporcional con las seis distintas dimensiones y las tareas propuestas para evaluar el razonamiento proporcional.

En lo referente a los niveles de logro y a las estrategias de resolución de problemas, muestran que los estudiantes son capaces de comprender diversas ideas del concepto de proporcionalidad y demuestra que el razonamiento proporcional es una ruta viable para acceder a las ideas poderosas en matemáticas.

A pesar de que los estudiantes no han desarrollado aún todas las estructuras cognitivas y matemáticas para comprender la complejidad del pensamiento proporcional, diversos estudios han permitido dilucidar etapas tempranas en el desarrollo cognitivo de los niños con tareas y enfoques pertinentes y muchos otros aspectos que influencian el desempeño de los estudiantes en las tareas.

\section{Agradecimientos}

Este artículo contó con el apoyo del proyecto de investigación SEP-CONACYT número 145906. Titulado: Introducción temprana al pensamiento algebraico en entornos tecnológicos de aprendizaje: un estudio teórico-experimental en el nivel básico, cuya responsable técnica fue la Dra. Cristianne Butto Zarzar.

\section{Referencias}

Bazán Ramirez, A., Sanchéz Hernández., B., \& Castañeda Figueiras, S. (2007). Relación estructural entre apoyo familiar, nivel educativo de los padres, características del maestro y desempeño en lengua escrita. Revista Mexicana de Investigación Educativa,12(33), p.p. 701 729.

Bazán Ramirez, A., Sanchéz Hernández., B., Corral Verdugo, V., \& Castañeda Figueiras, S. (2006).Utilidad de los modelos estructurales en el estudio de la lectura y la escritura. Revista Interamericana de Psicologia,40(1) p.p 85-94.

Butto, C. (2005). Introducción temprana al pensamiento algebraico: una experiencia en la escuela primaria. Tesis doctoral inédita, Cinvestavipn, México.

Carraher, T., Schliemann, A. y Carraher, D. (1985). Proporcionalidade na educaçao científica e matemática: uma análise de tarefas piagetianas. Revista Brasileira de Estudos Pedagógicos. 67(156). 367379.

Carraher, T., Schliemann, A., Carraher, D y Ruiz, E. (1986). Proporcionalidade na educacao científica e matemática: quantitadades, medidas por razoes. Revista Brasileira de Estudos Pedagógicos. 67(155). 367-379.

Díaz de León, Soto y Martínez (2007). Razonamiento proporcional intuitivo en alumnos de primaria y secundaria. Revista Interamericana de Psicología 41(3). 371-378

English, L. y Halford, G. (1995). Mathematics Education: models and processes. Lawrences Unite Kindong: Lawrence Earlbaun Associates.

Fernández, C. y Llinares, S. (2012). Características del desarrollo del razonamiento proporcional en la educación primaria y secundaria. Enseñanza de las Ciencias. 30 (1), 129-142.

Gómez, H. (1996), Indicios de pensamiento proporcional. Un estudio en la escuela primaria sobre competencias al resolver situaciones de cambio. Tesis de maestría. Departamento de matemática educativa, CINVESTAV-IPN, México.

Inhelder, B y Piaget, J. (1958). The Growth of Logical Thinking from Childhood to Adolescence, Nueva York, Basic Bools.

Karplus, R. y Peterson, R. (1970). Intellectual Development Beyond Elementary School II: Ratio, A Survey. Science, Curriculum Improvement Study. University of California.

Karplus, R., Pulus, S. y Stage, E. (1983). Proportional reasoning of early adolescents. En R. Lesh, \& M. Landau (Eds.), Acquisition of Mathematics concepts and processes.

Lunzer, E., Harrison, C. y Davey, M. (1972). The four-card problem and the development of formal reasoning. Quarterly Journal of Experimente Psycgology. 24. Pp. 326-339. 


\section{El razonamiento proporcional en educación básica}

Lunzer, E. Y Pumfrey, P. (1966). Understanding proportionality. Mathematics Teaching

Noelting, G. (1980). The develoment of proportional reasoning and the ratio concept: part I Diferentation of Stages. Educational Estudies in Mathematics. 11. P.p 217-253.

Obando, G., Vasco, C. y Arboleda, L. (2014). Enseñanza y aprendizaje de la razón, la proporción y la proporcionalidad: un estado del arte. Revista latinoamericana de investigación en matemática educativa. 17(1). 59-81.

Secretaría de Educación Pública (2011). Planes y programas de estudio. México, D.F.: Secretaria de Educación Pública.

Secretaría de Educación Pública (2011). Planes y programas de estudio. México, D.F.: Secretaria de Educación Pública.

Simce (2007). Niveles de logro 4o Básico, Lectura y educación matemática. Gobierno de Chile: Ministerio de Educación.
Spinillo, A. G. (1990). The development of the concept of proportion in Young children. Tese de Doctored. University of Oxford. Inglaterra.

Spinillo, A. G. \& Bryant, P. E. (1991). Children's proportional judgments: The importance of "half". Chil development. 62 427-440.

Spinillo, A. G. \& Bryant, P. E. (1993). Julgamentos proporcionais em crianças: comparando o desempeño: comparando o desempenho e as justificativas em tarefas numéricas e não-numéricas. 45 Reunião Anual da sociedade Brasileira para o progresso da Ciência.

Tourniaire, F. y Pulus, S.(1985). In Proportional Reasoning: A review of the literature Educational Studies in Mathematics. 16 pp181-204. Reidel Publishing Company. 